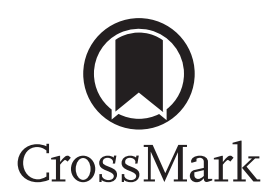

\title{
Quantifying comorbidity in individuals with COPD: a population study
}

\author{
Andrea S. Gershon 1,2,3,4, Graham C. Mecredy ${ }^{2}$, Jun Guan², J. Charles Victor ${ }^{2,3}$, \\ Roger Goldstein ${ }^{3,5}$ and Teresa To $2,3,4$
}

Affiliations: 'Sunnybrook Health Sciences Centre, Toronto, ON, Canada. ${ }^{2}$ Institute for Clinical Evaluative Sciences, Toronto, ON, Canada. ${ }^{3}$ University of Toronto, Toronto, ON, Canada. ${ }^{4}$ The Hospital for Sick Children, Toronto, ON, Canada. ${ }^{5}$ Westpark Healthcare Centre, Toronto, ON, Canada.

Correspondence: Andrea S. Gershon, Institute for Clinical Evaluative Sciences, G1 06, 2075 Bayview Avenue, Toronto, Ontario, M4N 3M5, Canada. E-mail: andrea.gershondices.on.ca

ABSTRACT Chronic obstructive pulmonary disease (COPD) has been associated with many types of comorbidity. We aimed to quantify the real world impact of COPD on lower respiratory tract infection, cardiovascular disease, diabetes, psychiatric disease, musculoskeletal disease and cancer, and their impact on COPD through health services.

A population study using health administrative data from Ontario, Canada, in 2008-2012 was conducted. Absolute and adjusted relative rates of ambulatory care visits, emergency department visits and hospitalisations for the comorbidities of interest in people with and without COPD were determined and compared.

Among 7241591 adults, 909948 (12.6\%) had COPD. Over half of all lung cancer, a third of all lower respiratory tract infection and cardiovascular disease, a quarter of all low trauma fracture, and a fifth of all psychiatric, musculoskeletal, non-lung cancer and diabetes ambulatory care visits, emergency department visits and hospitalisations in Ontario were used by people with COPD. Individuals with COPD used about five times more health services for lung cancer, and two times more health services for lower respiratory tract infections and cardiovascular disease than people without COPD.

Individuals with COPD use a disproportionate amount of health services for comorbid disease, placing significant burden on the healthcare system.

@ERSpublications

COPD responsible for significant proportion of all hospitalisations, emergency department visits and ambulatory care http://ow.ly/xVZMZ

For editorial comments see Eur Respir J 2015; 45: 14-17 [DOI: 10.1183/09031936.00148014].

This article has supplementary material available from erj.ersjournals.com

Received: April 012014 | Accepted after revision: June 062014 | First published online: August 202014

Support statement: Andrea Gershon is currently supported by a Fellowship in Translational Research from The Physicians' Services Incorporated Foundation, Toronto, Canada, and was supported by a New Investigator Award funded by team grant OTG-88591 from the Canadian Institutes of Health Research while working on this study. Teresa To is supported by The Dales Award in Medical Research from the University of Toronto, Toronto. Funding for this project was made available through the Government of Ontario, which had no role in study design, collection, management, analysis, interpretation of data, writing of the report or in the decision to submit the report for publication. This study was also supported by the Institute for Clinical Evaluative Sciences (ICES), which is funded by an annual grant from the Ontario Ministry of Health and Long-Term Care. The opinions, results and conclusions reported in this paper are those of the authors, and are independent from the funding sources. No endorsement by ICES or any of the funding agencies is intended or should be inferred.

Conflict of interest: None declared.

Copyright OERS 2015 


\section{Introduction}

Chronic obstructive pulmonary disease (COPD), a common, manageable lung disease, has much comorbidity $[1,2]$ that has significant impact on individuals and healthcare systems [3-6]. Treating this comorbidity improves people's overall health and may improve their COPD as well [1].

Associations between COPD and lower respiratory tract infection, cardiovascular disease, psychiatric disease, musculoskeletal disease, cancer and diabetes have been documented [7-13]. The magnitude of the real-world impact of COPD on each of these and their impact on COPD, however, has not been well quantified. Knowing how much these other diseases are affected by COPD would reveal whether COPD should be of concern not only to pulmonologists but to other specialists as well. Alternatively, knowing how much each of these other diseases impact people with COPD would help anticipate and prioritise an approach to COPD comorbidity.

To fill these knowledge gaps, we studied health services used by a large, real-world, multiethnic North American population using health administrative data. To address the first gap, we quantified what proportions of all hospitalisations, emergency department visits and ambulatory care visits for the comorbidities mentioned were made by people with COPD. To address the second gap, we quantified the incremental need for health services for each by people with COPD over people without COPD.

\section{Methods}

We conducted a longitudinal, population cohort study using universal health administrative data from Ontario, a province of Canada with a diverse multicultural population of approximately 13 million. Research ethics approval was obtained from the institutional review board at Sunnybrook Health Sciences Centre, Toronto, Ontario.

\section{Data sources}

Residents of Ontario have universal public health insurance under the Ontario Health Insurance Plan, the single payer for all medically necessary services across the full spectrum of residents, providers and hospitals. Service details are captured in health administrative databases that can be linked on an individual level to provide a complete health service profile for each resident. The only exception is the provision of prescription medications, which are only provided to those aged 65 years or older and those who are on welfare. Five Ontario population health-administrative databases were used: 1) the Registered Persons Database, which contains basic demographic information and date of death; the Canadian Institute of Health Information's 2) Discharge Abstract and 3) National Ambulatory Care Reporting System databases, which contain information on all discharges from acute care hospitals, same-day surgical procedures and emergency department visits; 4) the Ontario Health Insurance Plan Physician Claims database, which contains information about all services provided by fee-for-service physicians and "shadow billings" for physicians paid under alternate payment plans; and 5) the Ontario Mental Health Reporting System database, which contains information on patients in adult designated mental health beds.

\section{Subjects}

All individuals aged 35 years and older living in Ontario identified with physician-diagnosed COPD between April 1, 1991 and on March 31, 2008 were followed for 4 years. Those who died or left the province were censored.

\section{Exposure}

Individuals were identified as having physician-diagnosed COPD if they met a previously validated case definition that consisted of being aged 35 years or older, and having one previous COPD hospitalisation and/or ambulatory care visit as per International Classification of Disease (ICD) ninth revision codes 491, 492, and 496, and ICD-10 codes J41 to J44 [14]. This case definition had a sensitivity of $85.0 \%$ and a specificity of $78.4 \%$ when compared with real-world clinical evaluation by a physician. More details and examples of its use can be found elsewhere $[14,15]$. Because COPD is a disease with an insidious onset, those newly diagnosed with COPD in the follow-up period most likely had it in 2008, therefore they were also identified as having COPD.

\section{Outcomes}

The primary outcomes were rates of lower respiratory tract infection, cardiovascular disease, musculoskeletal disease, psychiatric disease, cancer and diabetes mellitus. Lower respiratory tract infection included pneumonia, influenza and acute bronchitis with or without a documented concurrent exacerbation of COPD. Musculoskeletal disease was divided into low trauma fractures, a previously used marker of osteoporosis, and other musculoskeletal disease. Cancers were divided into lung cancer and non-lung cancer. Ambulatory care visits, emergency department visits and hospitalisations for these 
comorbidities were determined from health-administrative data using appropriate ICD-9 and ICD-10 codes (online supplementary table S1). As it was possible for multiple ambulatory care claims for the same disease to occur during one day (i.e. one for diagnosis, one for laboratory investigations and one for imaging), only one ambulatory care claim per physician per patient per day and per disease was used to avoid over-counting. Since, as is reflective of the real world, people admitted to the hospital or emergency department could have had more than one condition and diagnostic code, a single admission could have been considered for multiple outcomes. For example, if a patient was admitted to hospital with diagnoses of both cardiovascular disease and a lower respiratory tract infection, the admission was counted once for each diagnosis.

\section{Covariates}

As characteristics of people with COPD generally differ from those of people without COPD, several potential confounding variables were considered when comparing health service use between these groups (table 1). Socioeconomic status was inferred from neighbourhood income derived from postal codes and census data [16]. Rural status was based on Statistics Canada's definition of rurality [17]. Asthma was also considered because it could potentially be misclassified as COPD [18]. Finally, analyses for each comorbidity were adjusted for all the other comorbidities of interest.

\section{Analysis}

t-tests and chi-squared tests were used to compare continuous and categorical characteristics, respectively, between individuals with and without COPD. Proportions of all hospitalisations, emergency department visits and ambulatory care visits for each comorbidity made by people with COPD in Ontario were determined.

Crude rates of claims per 1000 person-years and 95\% confidence intervals were calculated using the gamma distribution for each comorbidity [19].

In each healthcare domain, the adjusted relative rate of each comorbidity in those with compared to those without COPD adjusting for all covariates was estimated using a generalised linear model with Poisson distribution adjusted for overdispersion. All analyses were performed using SAS 9.1 software (SAS Institute, Cary, NC, USA).

\section{Sensitivity analyses}

To remove further the possibility of confounding by age, we stratified by younger (35 to 64 years) and older (65 years and older) adults. We also studied these age groups because managing COPD in each is a little different and we thought it would be of interest to clinicians. To determine if hospitalisation, emergency department visit and ambulatory care visit rates were being driven by a few high users of these services, we calculated their rates per 1000 patients instead of per 1000 person-years.

To assess if it was plausible that potential COPD misclassification was responsible for the observed results, a "true" relative rate based on the observed relative rate and the level of potential bias associated with misclassification was calculated [20]. We varied the theoretical relative rate of having a COPD comorbidity

\begin{tabular}{|c|c|c|c|}
\hline Characteristic & COPD population & Non-COPD population & p-value $\#$ \\
\hline Subjects n $(\%)$ & $909948(12.6)$ & $6331643(87.4)$ & \\
\hline Age years mean $\pm s D$ & $64.3 \pm 13.8$ & $53.4 \pm 13.3$ & $<0.001$ \\
\hline Females & 50.4 & 52.2 & $<0.001$ \\
\hline \multicolumn{4}{|l|}{ Socioeconomic status } \\
\hline Quintile 1T & 23.4 & 18.0 & $<0.001$ \\
\hline Quintile 2 & 21.8 & 19.4 & \\
\hline Quintile 3 & 19.4 & 19.9 & \\
\hline Quintile 4 & 18.4 & 21.0 & \\
\hline Quintile $5^{+}$ & 16.7 & 21.5 & \\
\hline Rural $^{\S}$ & 16.2 & 11.9 & $<0.001$ \\
\hline Asthma & 26.9 & 8.3 & $<0.001$ \\
\hline Deaths $^{f}$ & 14.6 & 3.3 & $<0.001$ \\
\hline
\end{tabular}

\#: testing the hypothesis of no difference between the COPD and non-COPD populations; ": lowest; ${ }^{+}$: highest; ${ }^{\S}$ : versus urban; ${ }^{f}$ : accumulated mortality rate at 4 years. 
between those who were potentially misclassified (based on a previous validation study [14]) and those who were correctly classified to see at what point the observed relative rate was rendered null (i.e. with a $95 \%$ confidence interval that included 1.00 ).

\section{Results}

Subjects

There were 7241591 residents of Ontario age 35 years and older on April 1, 2008, of whom 731717 had physician-diagnosed COPD. The 178231 individuals diagnosed with COPD during the follow-up period were also presumed to have COPD, for a total of 909948 (12.6\%) individuals (fig. 1). Individuals with COPD were older and more likely to be male, of lower socioeconomic status, live in a rural area and have asthma (table 1).

\section{Proportions of health services used by people with COPD}

Over half of all lung cancer, a third of all lower respiratory tract infection and cardiovascular disease, a quarter of all low trauma fracture, and a fifth of all psychiatric, musculoskeletal, non-lung cancer and diabetes health services in Ontario were used by people with COPD (fig. 2). Over 60\% of lower respiratory tract infection and lung cancer hospitalisations and over $40 \%$ of cardiovascular hospitalisations were made by people with COPD.

\section{Crude rates}

Comorbidities with the highest crude claim rates were psychiatric disease for ambulatory care visits, diabetes for emergency department visits and cardiovascular disease for hospitalisations (table 2).

\section{Adjusted relative rates}

Individuals with COPD had significantly higher adjusted relative rates of health service use for all the comorbidities compared to the general population. Rates of hospitalisation for lower respiratory tract infection and all rates for lung cancer were roughly five times greater than in the rest of the population. The rest of the rates for lower respiratory tract infection, and rates of emergency department visits and hospitalisations for cardiovascular, psychiatric disease and diabetes in people with COPD were about double the rates of the rest of the population (fig. 3).

\section{Sensitivity analyses}

$51 \%$ of individuals with COPD were between 35 and 64 years. Comparative demographics between people with and without COPD in these cohorts were similar to the overall population. As expected, compared

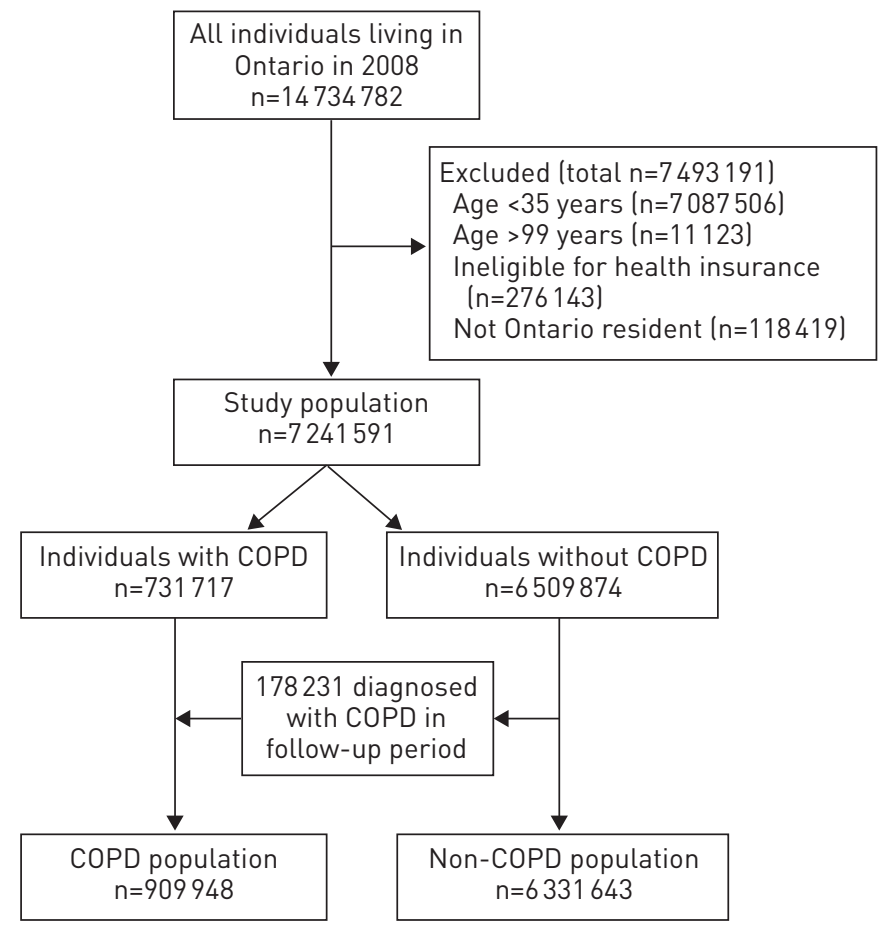

FIGURE 1 Flow chart of study subjects. COPD: chronic obstructive pulmonary disease. 


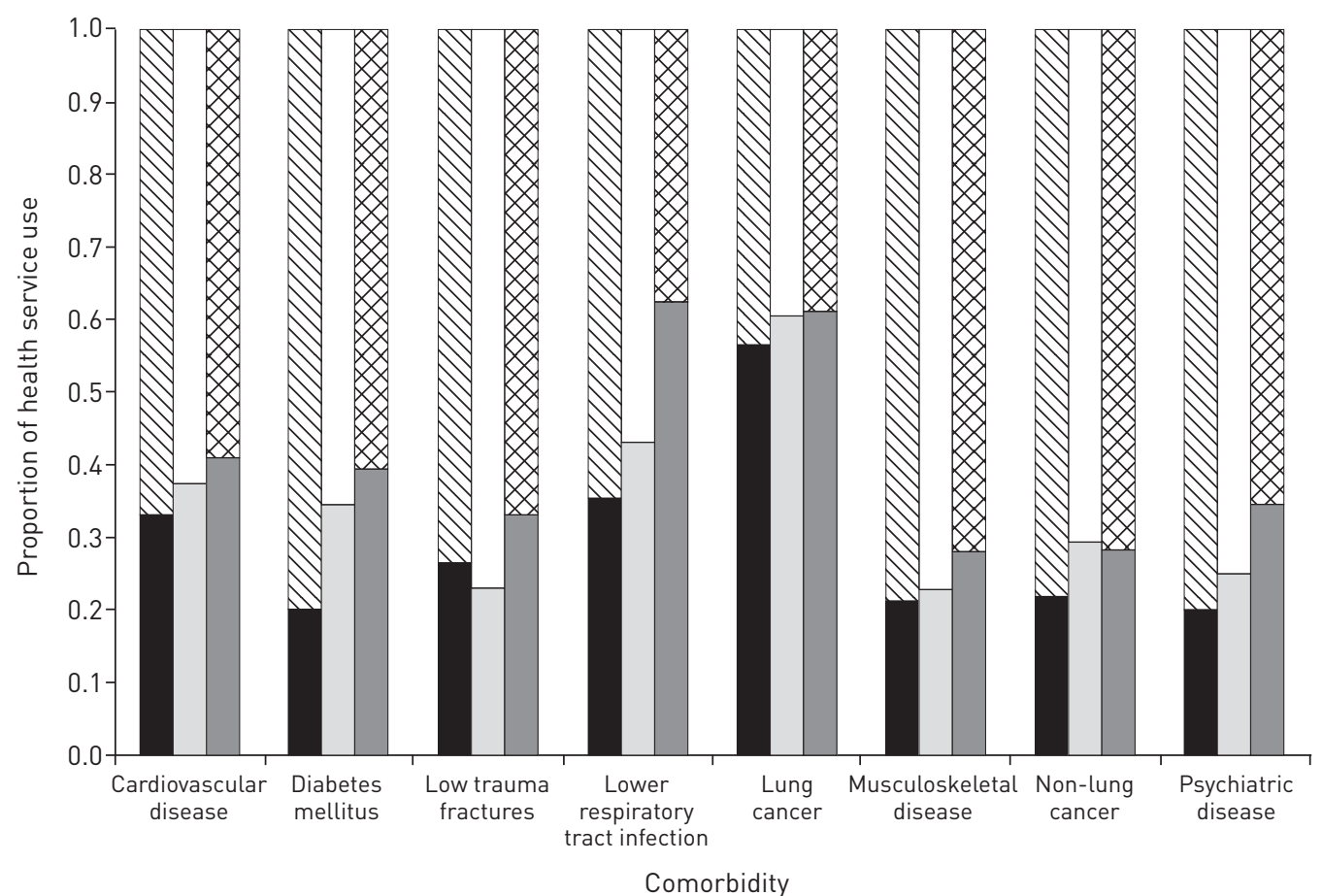

\footnotetext{
Health service use in people with COPD

Ambulatory care visits

$\square$ Emergency department visits

$\square$ Hospitalisations
}

Health service use in people without COPD

$\triangle$ Ambulatory care visits

$\square$ Emergency department visits

$叉$ Hospitalisations

FIGURE 2 Proportion of all ambulatory care visits, emergency department visits and hospitalisations made in Ontario by individuals with chronic obstructive pulmonary disease (COPD) for various types of COPD comorbidity. Overall prevalence of COPD is $12.6 \%$.

with younger individuals, older individuals had higher crude rates of health service use for all comorbidities except psychiatric disease ambulatory care and emergency department visits (tables S2 and S3). Furthermore, older individuals with COPD used a higher proportion of all health services for all comorbidities than their younger counterparts (figs S1 and S2). However, younger individuals had higher adjusted relative rates of almost all comorbidities across all health services compared with older individuals (fig. S3).

When measuring claims per 1000 patients instead of person-years, proportions of people with health service claims for each comorbidity and adjusted relative rates were lower but still notable (table S4, and figs S4 and S5).

As COPD increased the risk of having comorbid disease, anyone misclassified in our COPD population (i.e. who did not actually have COPD) probably had a lower risk of comorbidity, thus biasing our findings towards the null. Therefore, when potential misclassification was accounted for, the observed relative rates became even greater (table S5). Finally, even if those misclassified had an increased risk of developing comorbidity, our results would not easily be rendered null (table S5).

\section{Discussion}

We studied a large, real-world, North American COPD population using health administrative data and found that individuals with COPD, who comprised $12.6 \%$ of the population, were responsible for over half of all lung cancer, a third of all lower respiratory tract infection and cardiovascular disease, a quarter of all low trauma fracture, and a fifth of all psychiatric, musculoskeletal, non-lung cancer and diabetes hospitalisations, emergency department visits and ambulatory care visits. Furthermore, individuals with COPD used roughly five times more health services for lung cancer and two times more health services for lower respiratory tract infection and cardiovascular disease than people without COPD. To the best of our knowledge, this is the first large-scale, real-world study to quantify the impact of different types of COPD comorbidity in terms of health service use and measure the overall impact of COPD on other diseases. This new knowledge emphasises the need to both consider COPD when managing other diseases and address other diseases when managing people with COPD. 
TABLE 2 Crude number of health services claims and claim rate for chronic obstructive pulmonary disease (COPD) and various types of COPD comorbidity in individuals with COPD

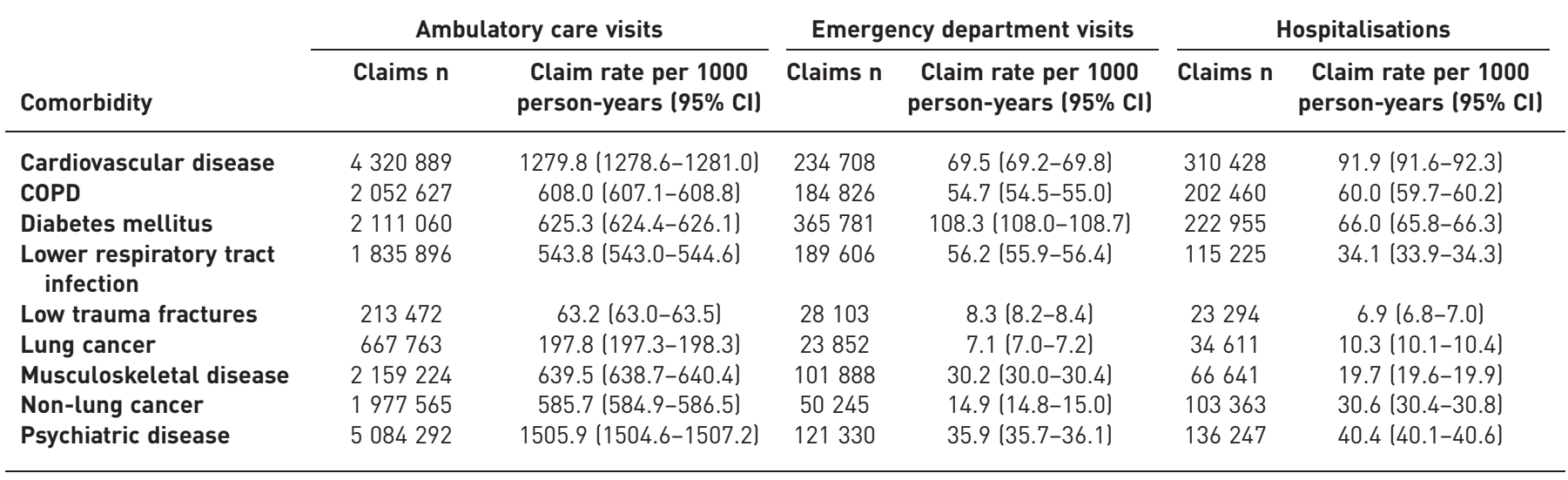

Our findings are consistent with previous population-based studies demonstrating an association between COPD and a wide range of comorbidities [13, 21-29]. For example, GARCIA-RodriguEz et al. [23] found an increased risk of lung cancer (relative risk 3.33, 95\% CI 2.33-4.75) and heart failure (relative risk 2.94, 95\% CI 2.46-3.51), while NG et al. [27] found an increased risk of depressive symptoms (OR 1.86, 95\% CI 1.25-2.75) among those with COPD compared with those without. Our findings are also consistent with our previous study demonstrating that a large proportion of the additional health services used by individuals with COPD was to address COPD-attributable comorbidity [5]. Our study extends these previous findings by, first, demonstrating that a large proportion of health service use for several common diseases is by people with COPD and, second, by quantifying the increased need for health services for several major comorbidities in a large, complete, real-world COPD population. By doing so, it reveals a more practical and precise account of how COPD impacts individuals and society.

Several theories have been put forth to explain the overlap between COPD and the diseases that make up its comorbidity, including: a "spill over" of pulmonary inflammation into the systemic circulation to affect other areas of the body; shared genetic predispositions between COPD and other diseases; and common environmental exposures, the most notable being cigarette smoking $[1,2,30]$. Despite overlap, however, diagnosing and treating COPD comorbidity is not a standard part of COPD management, as evidenced by the little attention it receives in many COPD practice guidelines [31,32], although this is starting to change [33].

It is also likely that some of the association between increased health services use for comorbidity and COPD is because COPD lowers patients' ability to tolerate other diseases causing patients to require medical attention for them when they are at earlier stages. For example, people with COPD have impaired ventilatory reserve and, therefore, are more likely to require hospitalisation for pneumonia than those with healthy lungs.

In the stratified analyses, there were universally higher relative rates of COPD comorbidity among younger compared with older adults. This probably points to low rates of comorbidity in younger populations in general so that when younger COPD patients develop even minor comorbidity, the proportional increase is large (even if the absolute increase is small). This highlights the need to detect and address comorbidity to keep this younger age group healthy.

There were a couple of findings of interest in our study. One was that younger individuals had higher claim rates for psychiatric ambulatory and emergency department visits than older individuals. This is consistent with previous literature showing higher rates of psychiatric disorders among younger age groups compared with older ones in both the general and COPD population $[34,35]$. One possible explanation is that younger individuals, who do not anticipate illness as older individuals do, might be less able to cope with health-related stressors such as COPD. A second point of interest was that people with COPD had more health service claims for cardiovascular disease, diabetes and psychiatric disease than for COPD itself. Previously, our group has demonstrated relatively low rates of health services use for COPD in relation to its comorbidity in people with COPD [5] and we believe this points to the high rate of comorbidity in this population. In addition, rates of admission for COPD were reported separate from 

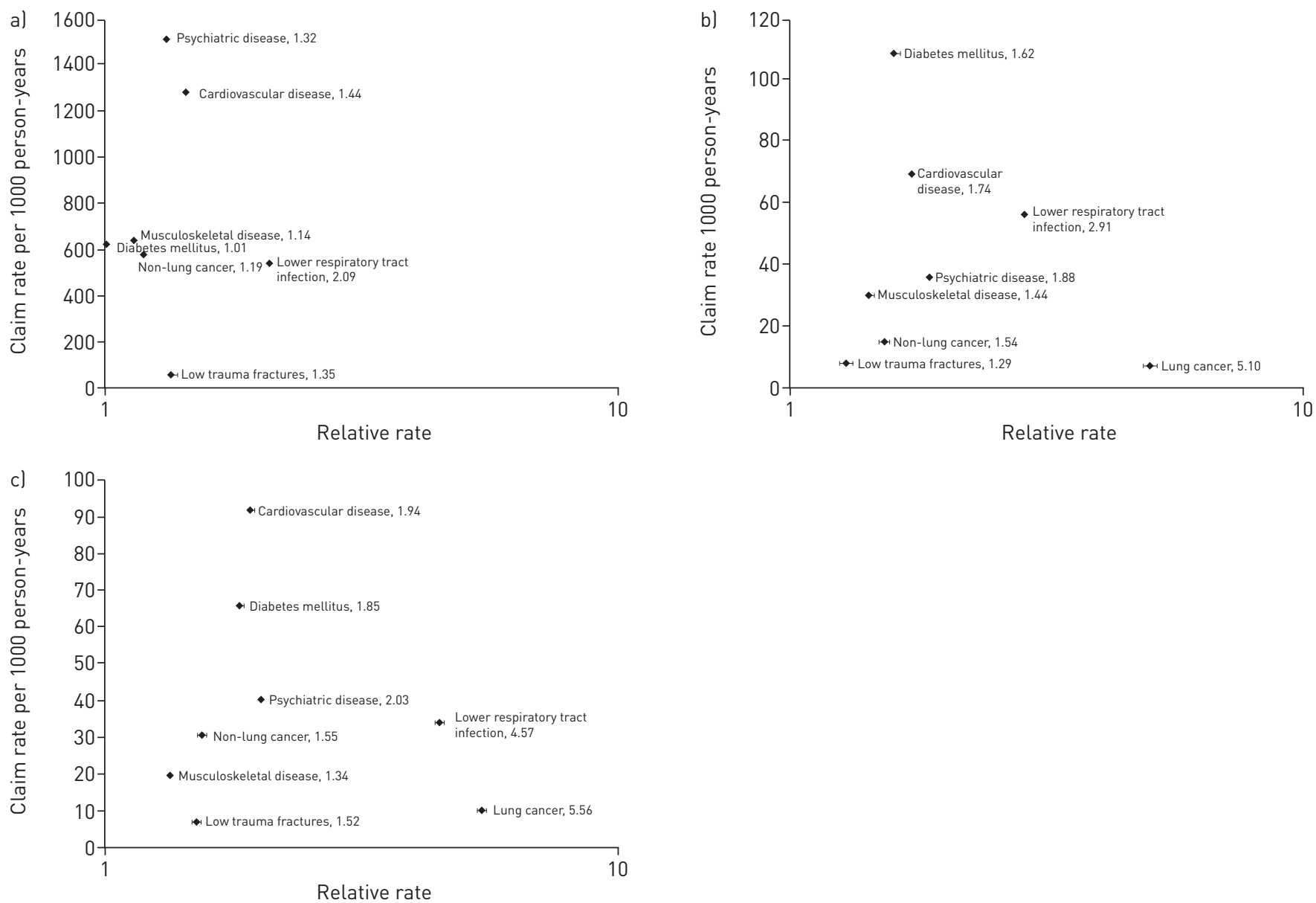

FIGURE 3 Relationship between claim rate per 1000 person-years of chronic obstructive pulmonary disease (COPD) comorbidities and their adjusted relative rates (with 95\% confidence intervals) of a) ambulatory care visits, b) emergency department visits and c) hospitalisations in individuals with COPD compared with those without COPD. Adjusted for age, sex, income quintile, rurality, asthma diagnosis and all other comorbidities. Confidence intervals may not be visible due to their small ranges.

rates of lower respiratory tract infections when, in reality, both categories together would constitute acute exacerbations of COPD. If this were taken into consideration, the rate of COPD health service use would have approached or exceeded that of those other diseases.

The strengths of our study are its use of a complete, real-world population and ability to capture and quantify health service use for several different diseases. In addition, because all individuals were covered by universal healthcare, there is minimal concern of bias due to financial barriers to care. It also has limitations that merit emphasis. First, even though our health administrative case-definition has been validated [14], some individuals with physician-diagnosed COPD may have been misclassified as non-COPD in our study, and vice versa. Overall, however, since COPD is generally underdiagnosed by physicians, we believe our absolute measures likely underestimate true COPD [36, 37]. In addition, because our relative measures of health service use were based on differences between individuals with and without COPD, misclassification would have caused them to be underestimates also. To address this further, however, we conducted a sensitivity analysis that showed that even in the unlikely scenario where all misclassified with COPD had another condition with a rate of comorbidity $50 \%$ higher than those with true COPD, our observed adjusted relative rates associated with COPD would not be negated (table S5). More realistically, individuals misclassified as having COPD probably had asthma, which is not associated with such high health services use, meaning our observed relative rates are probably underestimates.

A second limitation is that our case definition of COPD did not include spirometry and, as such, it identified physician-diagnosed COPD but not spirometry-confirmed COPD. This was done because we wanted to capture information about an entire population of people with physician-diagnosed COPD and only about $30 \%$ to $50 \%$ of people with COPD are diagnosed with spirometry [38]. Thus, our study is reflective of real-world practise and physician-diagnosed COPD. It only approximates, however, true COPD diagnoses. 
A third important limitation is the generalisability of the results. While studying a population with universal healthcare coverage may minimise bias due to financial barriers, it also might underestimate the burden of COPD comorbidity in jurisdictions without universal coverage, such as the USA. This is because uninsured patients in these jurisdictions are less likely to use ambulatory care services and homecare because of their cost, and as a result are more likely to require costly acute health services and die. Because of this, and since similarities between healthcare in Canada and other westernised countries greatly outweigh their differences, our results should be of concern to healthcare providers and decision makers in jurisdictions without universal healthcare insurance as well [5].

A fourth limitation is that our identification of disease depends on health service use, so people who use more health services are more likely to be diagnosed with COPD as well as other comorbid diseases. These higher volume health system users are also likely to have moderate to severe disease. Therefore, our study might have missed some individuals with milder disease.

\section{Conclusion}

People with COPD are responsible for a large and disproportionate amount of health service use for many comorbidities. Future investigation should characterise what the impact of comorbidities is on hospitalisations, emergency department visits and ambulatory care for COPD, and vice versa. For example, for hospitalisation, it would be of interest to know the impact on risk of death, length of stay and disposition. Through better understanding of the complex interactions between COPD and its comorbidity we can optimise patient care and improve health outcomes.

\section{References}

1 Decramer M, Janssens W. Chronic obstructive pulmonary disease and comorbidities. Lancet Respir Med 2013; 1: $73-83$.

2 Agusti A, Faner R. Systemic inflammation and comorbidities in chronic obstructive pulmonary disease. Proc Am Thorac Soc 2012; 9: 43-46.

3 Burgel PR, Escamilla R, Perez T, et al. Impact of comorbidities on COPD-specific health-related quality of life. Respir Med 2012; 107: 233-241.

4 Divo M, Cote C, de Torres JP, et al. Comorbidities and risk of mortality in patients with chronic obstructive pulmonary disease. Am J Respir Crit Care Med 2012; 186: 155-161.

5 Gershon AS, Guan J, Victor JC, et al. Quantifying health services use for chronic obstructive pulmonary disease. Am J Respir Crit Care Med 2013; 187: 596-601.

6 Liapikou A, Polverino E, Ewig S, et al. Severity and outcomes of hospitalised community-acquired pneumonia in COPD patients. Eur Respir J 2012; 39: 855-861.

$7 \quad$ Rabe KF, Wedzicha JA, Wouters EFM. COPD and Comorbidity. ERS Monogr 2013; 59.

8 Boutin-Forzano S, Moreau D, Kalaboka S, et al. Reported prevalence and co-morbidity of asthma, chronic bronchitis and emphysema: a pan-European estimation. Int J Tuberc Lung Dis 2007; 11: 695-702.

9 Feary JR, Rodrigues LC, Smith CJ, et al. Prevalence of major comorbidities in subjects with COPD and incidence of myocardial infarction and stroke: a comprehensive analysis using data from primary care. Thorax 2010; 65: 956-962.

10 Li L, Brennan KJ, Gaughan JP, et al. African Americans and men with severe COPD have a high prevalence of osteoporosis. COPD 2008; 5: 291-297.

11 Mannino DM, Thorn D, Swensen A, et al. Prevalence and outcomes of diabetes, hypertension and cardiovascular disease in COPD. Eur Respir J 2008; 32: 962-969.

12 Sethi S. Infection as a comorbidity of COPD. Eur Respir J 2010; 35: 1209-1215.

13 Soriano JB, Visick GT, Muellerova H, et al. Patterns of comorbidities in newly diagnosed COPD and asthma in primary care. Chest 2005; 128: 2099-2107.

14 Gershon AS, Wang C, Guan J, et al. Identifying individuals with physician diagnosed COPD in health administrative databases. J COPD 2009; 6: 388-394.

15 Gershon AS, Warner L, Cascagnette P, et al. Lifetime risk of developing chronic obstructive pulmonary disease (COPD). Lancet 2011; 378: 991-996.

16 PCCF+ Version 4D User's Guide (Geocodes/PCCF). Automated Geographic Coding Based on the Statistics Canada Postal Code Conversion Files, Including Postal Codes to December 2003. Ottawa, Statistics Canada, 2004.

17 Plessis V, Beshiri R, Bollman R, et al. Definitions of "Rural". Ottawa, Statistics Canada, 2002

18 Gershon AS, Wang C, Vasilevska-Ristovska J, et al. Identifying patients with physician diagnosed asthma in health administrative databases. Can Respir J 2009; 16: 183-188.

19 Fay MP, Feuer EJ. Confidence intervals for directly standardized rates: a method based on the gamma distribution. Stat Med 1997; 16: 791-801.

20 Schneeweiss S. Sensitivity analysis and external adjustment for unmeasured confounders in epidemiologic database studies of therapeutics. Pharmacoepidemiol Drug Saf 2006; 15: 291-303.

21 Barr RG, Celli BR, Mannino DM, et al. Comorbidities, patient knowledge, and disease management in a national sample of patients with COPD. Am J Med 2009; 122: 348-355.

22 Garcia-Olmos L, Alberquilla A, Ayala V, et al. Comorbidity in patients with chronic obstructive pulmonary disease in family practice: a cross sectional study. BMC Fam Pract 2013; 14: 11.

23 Garcia Rodriguez LA, Wallander MA, Martin-Merino E, et al. Heart failure, myocardial infarction, lung cancer and death in COPD patients: a UK primary care study. Respir Med 2010; 104: 1691-1699.

24 Joo H, Park J, Lee SD, et al. Comorbidities of chronic obstructive pulmonary disease in Koreans: a population-based study. J Korean Med Sci 2012; 27: 901-906. 
25 Kornum JB, Svaerke C, Thomsen RW, et al. Chronic obstructive pulmonary disease and cancer risk: a Danish nationwide cohort study. Respir Med 2012; 106: 845-852.

26 Lin PJ, Shaya FT, Scharf SM. Economic implications of comorbid conditions among Medicaid beneficiaries with COPD. Respir Med 2010; 104: 697-704.

27 Ng TP, Niti M, Fones C, et al. Co-morbid association of depression and COPD: a population-based study. Respir Med 2009; 103: 895-901.

28 Schneider C, Bothner U, Jick SS, et al. Chronic obstructive pulmonary disease and the risk of cardiovascular diseases. Eur J Epidemiol 2010; 25: 253-260.

29 Schnell K, Weiss CO, Lee T, et al. The prevalence of clinically-relevant comorbid conditions in patients with physician-diagnosed COPD: a cross-sectional study using data from NHANES 1999-2008. BMC Pulm Med 2012; 12: 26 .

30 Sinden NJ, Stockley RA. Systemic inflammation and comorbidity in COPD: a result of overspill of inflammatory mediators from the lungs? Review of the evidence. Thorax 2010; 65: 930-936.

31 Qaseem A, Wilt TJ, Weinberger SE, et al. Diagnosis and management of stable chronic obstructive pulmonary disease: a clinical practice guideline update from the American College of Physicians, American College of Chest Physicians, American Thoracic Society, and European Respiratory Society. Ann Intern Med 2011; 155: 179-191.

32 O'Donnell DE, Hernandez P, Kaplan A, et al. Canadian Thoracic Society recommendations for management of chronic obstructive pulmonary disease - 2008 update - highlights for primary care. Can Respir J 2008; 15: Suppl. A, $1 \mathrm{~A}-8 \mathrm{~A}$.

33 Global Initiative for Chronic Obstructive Lung Disease. Global strategy for the diagnosis, management and prevention of chronic obstructive pulmonary disease. GOLD, 2013.

34 Cleland JA, Lee AJ, Hall S. Associations of depression and anxiety with gender, age, health-related quality of life and symptoms in primary care COPD patients. Fam Pract 2007; 24: 217-223.

35 Offord DR, Boyle MH, Campbell D, et al. One-year prevalence of psychiatric disorder in Ontarians 15 to 64 years of age. Can J Psychiatry 1996; 41: 559-563.

36 Buist AS, McBurnie MA, Vollmer WM, et al. International variation in the prevalence of COPD (the BOLD Study): a population-based prevalence study. Lancet 2007; 370: 741-750.

37 Halbert RJ, Natoli JL, Gano A, et al. Global burden of COPD: systematic review and meta-analysis. Eur Respir J 2006; 28: 523-532.

38 Gershon AS, Hwee J, Croxford R, et al. Patient and physician factors associated with pulmonary function testing for COPD: a population study. Chest 2014; 145: 272-281. 